\title{
Fibrosis quística: enfrentando la transición desde el pediatra hacia el internista
}

\author{
Luis E Vega-Briceñoํㅗㄹ \\ Ignacio Sánchez $D^{1}$. \\ Cystic fibrosis: \\ facing patient transition \\ from the pediatrician to the internist
}

\begin{abstract}
The increased survival of patients with cystic fibrosis (CF) resulted in the appearance of new pulmonary and non-pulmonary complications. Even though subjects with $\mathrm{CF}$ present with inflammatory pulmonary changes at birth, several pathogens such as Staphyloccocus aureus, Haemophilus influenzae and Pseudomona aeruginosa contribute to the progression of pulmonary injury. Clinical presentations vary according to patient age; even though mild forms exist, patients with severe forms, develop respiratory insufficiency and end-stage disease at an early stage. Today, new diagnostic and therapeutic tools, increase the possibility of an early diagnosis and of greater survival. Successful management will depend on the timely selection of adequate antimicrobials, the use of pancreatic enzyme supplementation and early institution to respiratory physiotherapy. The transition from pediatric care to adult care must occur according to development of each patient. This timing must be flexible and there should be a constant communication and coordination within the different specialists in internal medicine. Thus, it is crucial to recognize the disease progression as a continuous process, giving the appropriate physiologic support and evaluating the needs of the patient and close relatives (Rev Méd Chile 2006; 134: 365-71).
\end{abstract}

(Key words: Cystic fibrosis; Respiratory insufficiency; Respiratory tract infectious)

Recibido el 22 de julio, 2005. Aceptado el 5 de septiembre, 2005.

${ }^{1}$ Secciones de Respiratorio Pediátrico y de ${ }^{2}$ Gastroenterología, Departamento de Pediatría, Pontificia Universidad Católica de Chile, Santiago de Chile.

$\mathrm{L}$ a transición de pacientes con enfermedades crónicas desde la niñez a la adultez implica desarrollar programas capacitados para una tran-

Correspondencia a: Dr. Luis Enrique Vega-Briceño. Laboratorio Respiratorio Pediátrico, Departamento de Pediatría, Pontificia Universidad Católica. Lira $855^{\circ}$ piso 114-D, Santiago Centro. Casilla postal 114-D. Teléfono: 354-3767. Fax: 247-3879. E mail: levega@puc.cl sición fluida y el control de pacientes cada vez más complejos ${ }^{1,2}$. El número de pacientes con fibrosis quística (FQ) que sobreviven y alcanzan la edad adulta ha mostrando un incremento significativo durante los últimos veinte años ${ }^{3}$; en algunos centros de FQ europeos, más de $40 \%$ de pacientes son adultos. Según cifras nacionales, hasta junio de 2005, en Chile existían al menos 309 pacientes con FQ registrados en el Programa Ministerial, de los cuales $44(14,2 \%)$ eran mayores 
de 18 años (comunicación personal). Si bien la sobrevida se ha incrementado, el número de complicaciones respiratorias y no respiratorias también ha mostrado un aumento significativo, siendo más frecuentes en la edad adulta ${ }^{3,4}$. Recientemente, la rama broncopulmonar de la Sociedad Chilena de Pediatría publicó las normas nacionales para estandarizar y optimizar el manejo de la FQ en niños 5 . El objetivo de la presente revisión es actualizar algunos aspectos de las manifestaciones clínicas en adolescentes y adultos jóvenes; brindando consideraciones para enfrentar la transición desde el niño hacia la adultez.

\section{EPIDEMIOLOGÍA}

La FQ es la enfermedad autosómica recesiva letal más frecuente en la raza caucásica. Tiene una evolución crónica, progresiva, con compromiso multisistémico y grandes variaciones fenotípicas entre individuos. Si bien es cierto, existe un incremento en el número de casos comunicados en Chile, el promedio de sobrevida aún no es ni siquiera la mitad de lo reportado en Estados
Unidos, donde se lo estima en 35 años, lo cual representa el doble de lo que fue hace 20 años $^{3,6}$. La incidencia de FQ en el hemisferio norte es $\sim 1 /$ 2.500 nacidos vivos, siendo $50 \%$ diagnosticados a la edad de 6 meses y $90 \%$ a la edad de 8 años ${ }^{6}$. En Chile, se estima una incidencia $\sim 1 / 6.000$ nacidos vivos. Nuestro grupo reportó una prevalencia de la mutación $\Delta \mathrm{F} 508$ cercana a 50\%, siendo la segunda mutación más frecuente la G542X. Otras mutaciones encontradas fueron: W1282X, R1162X, R553X, G551D, R334W y 3849+10kbC $>T^{7}$.

\section{Manifestaciones CLÍNICAS}

Las manifestaciones cardinales de la FQ varían según la edad del paciente; en general corresponden a una enfermedad pulmonar obstructiva crónica, insuficiencia pancreática exocrina y concentración elevada de electrolitos en el sudor. En más de la mitad de los pacientes con FQ, el diagnóstico se sospecha por la presencia de síntomas respiratorios. La Tabla 1 muestra las manifestaciones clínicas más frecuentes en escolares y adolescentes.

\section{Tabla 1. D istribución de síntomas y presentaciones clínicas más frecuentes} según la edad del paciente con fibrosis quística

\begin{tabular}{|c|}
\hline $\begin{array}{ll}\text { Escolares } \\
\text { - } & \text { Sintomas respiratorios crónicos sin causa evidente } \\
\text { - } & \text { Sinusitis crónica, poliposis nasal } \\
\text { - } & \text { Bronquiectasias } \\
\text { - } & \text { Diarrea crónica } \\
\text { - } & \text { Síndrome de obstrucción intestinal distal } \\
\text { - } & \text { Pancreatitis } \\
& \text { Prolapso rectal, hepatomegalia } \\
\text { Adolescentes y adultos } \\
\text { - } & \text { Enfermedad pulmonar supurativa crónica e inexplicada } \\
\text { - } & \text { Hipocratismo digital } \\
\text { - } & \text { Dolor abdominal recurrente } \\
\text { - } & \text { Pancreatitis, diabetes } \\
\text { - } & \text { Cíndrome de obstrucción intestinal distal } \\
\text { - } & \text { Retardo del crecimiento } \\
\text { - } & \text { Infertilidad masculina con azoospermia } \\
\text { - } & \text { Disminución de la fertilidad en mujeres }\end{array}$ \\
\hline
\end{tabular}


Manifestaciones y aspectos respiratorios. La enfermedad respiratoria es la responsable de la mayor morbimortalidad; más de 95\% de los pacientes con FQ presentan algún compromiso respiratorio, siendo más grave en los adolescentes y adultos ${ }^{4,8}$. En general, los pacientes adultos tienen cinco veces más prevalencia de compromiso pulmonar severo que los niños. Si bien el genotipo de CFTR es un buen predictor de la función exocrina del páncreas (homocigotos $\Delta \mathrm{F} 508$ tienen riesgo $\sim 100 \%$ de insuficiencia pancreática), se reconoce que existe una compleja relación genotipo-fenotipo con diversos grados de alteración en la función pulmonar ${ }^{9}$, por lo que las manifestaciones respiratorias varían ampliamente entre individuos afectados incluso con la misma mutación ${ }^{6,8}$, lo cual sugiere la presencia de otros factores genéticos o ambientales ${ }^{10,11}$.

En todo paciente con poliposis nasal sin una causa clara debe investigarse FQ, ya que 10-25\% de los pacientes con FQ la padecen. La aspergilosis broncopulmonar alérgica es un tipo de reacción alérgica infrecuente en la FQ (0,6-11\%), siendo más prevalente después de la primera década de la vida ${ }^{12,13}$. Su detección precoz requiere alta sospecha clínica y una búsqueda sistemática. La incidencia de bronquiectasias aumenta después de la segunda década de la vida, siendo la hemoptisis una de las formas frecuentes de presentación, la cual puede traducir una exacerbación aguda, déficit de vitamina K, progresión de enfermedad hepática (usualmente se manifiesta como hematemesis) o más raramente, efecto colateral de alguna droga. Cuando la hemoptisis es de gran magnitud ( $\geq 240 \mathrm{ml} /$ día, $\sim 6 \%$ de los pacientes con FQ) o recurrente, debe hospitalizarse el paciente, pudiendo requerirse la embolización de arterias bronquiales con un éxito $\sim 90 \% 14$. El neumotórax, producto de la ruptura de bulas subpleurales, tiene incidencia entre 3 y 19\%, siendo más frecuente en la adultez. Se asocia con una función pulmonar deteriorada y sugiere una enfermedad pulmonar grave. No existen preferencias por el lado afectado ni por sexo. Pueden ser pequeños o de gran tamaño asociados a dolor (50\%) y dificultad respiratoria (65\%); solo $16 \%$ son asintomáticos. Usualmente requieren la inserción de tubos de drenaje, con elevada tasa de fracaso (72\%), por lo que algunos autores recomiendan la pleurodesis temprana. El pronóstico es malo, con alta recurrencia y promedio de sobrevida de 30 meses luego del primer neumotórax espontáneo ${ }^{15}$.

La radiografía de tórax permite evaluar la progresión de la enfermedad pulmonar. Existen diversos puntajes de gravedad pero todos carecen de apropiada sensibilidad y especificidad ${ }^{12}$. La tomografía de alta resolución ha proporcionado una rápida y correcta evaluación pulmonar, siendo muy sensible y específica para determinar la localización de bronquiectasias, sugiriéndose que estos cambios podrían preceder a cambios de la función pulmonar ${ }^{16}$. Los pacientes con FQ usualmente presentan opacidad total de los senos paranasales por lo que debe considerarse el estudio con imágenes ${ }^{12}$. La determinación de la capacidad vital forzada (CVF), el volumen espirado forzado en el primer segundo $\left(\mathrm{VEF}_{1}\right)$ y la relación $\mathrm{VEF}_{1} / \mathrm{CVF}$ usualmente revelan un patrón obstructivo reversible a la administración de salbutamol. Sin embargo, esta respuesta es variable, dependiendo de la etapa evolutiva de la enferme$\mathrm{dad}^{5,12}$. La espirometría evalúa la efectividad del tratamiento y pesquisa precozmente algunas descompensaciones subclínicas. Se recomienda practicar la pletismografía, ya que algunos sujetos tienen volúmenes pulmonares anormales aun en presencia de una espirometría normal, siendo probablemente más precoces los cambios pletismográficos. En algunas situaciones debe considerarse la saturación arterial de oxígeno nocturna y la medición de gases arteriales para evaluar la necesidad de oxigenoterapia domiciliaria.

Aspectos gastrointestinales y nutricionales. Las manifestaciones clínicas son causadas por la incapacidad de los lúmenes de las glándulas de secreción exocrina de hidratar las macromoléculas producidas y transportarlas a través de ellas ${ }^{17}$. Las secreciones del páncreas y el intestino son espesas y viscosas, lo que lleva a obstrucción y frecuentemente a destrucción del tejido glandular con reemplazo de este por tejido fibroso. Las proenzimas digestivas pancreáticas, son retenidas en los ductos y activadas en forma prematura, lo que colabora con la destrucción. Se reconoce que esta transformación fibrosa se inicia en etapas tempranas de la vida, incluso desde el nacimiento ${ }^{8}$.

Aproximadamente, $85-90 \%$ de los pacientes con FQ tienen alterada la actividad de la lipasa pancreática. Para expresar insuficiencia pancreáti- 
ca exocrina, se necesita tener dos mutaciones severas $(\Delta \mathrm{F} 508$, W846X, 4005+1G->A, DeltaI507, S549R(T->G), E60X). Por otro lado, los pacientes con insuficiencia pancreática exocrina tienen tejido pancreático residual que puede promover una respuesta inflamatoria activa, siendo más susceptibles a desarrollar pancreatitis aguda recurrente o crónica $^{18}$. Progresivamente se pierden islotes de Langerhans, por lo que puede producirse manifestaciones de diabetes mellitus o intolerancia a la glucosa ( $\sim 30 \%$ de adolescentes y adultos con FQ). La diabetes mellitus en la FQ es la forma más frecuente de diabetes no autoinmune y suele aparecer en la segunda década de la vida ${ }^{19}$, por lo que se recomienda una evaluación anual de la tolerancia a glucosa luego de la pubertad ${ }^{20}$.

La desnutrición que sufren los pacientes con FQ exacerba el deterioro pulmonar. Es el resultado de una malabsorción de nutrientes, aumento del gasto energético, infecciones pulmonares recurrentes y una inadecuada ingesta calórica ${ }^{20,21}$. Por otro lado, existe la prescripción (errónea) de dietas pobres en grasa y por ello, en energía. El reflujo gastroesofágico ocurre en $20-25 \%$ de los pacientes y contribuye a causar mayor daño pulmonar y mal incremento ponderal ${ }^{22}$. Existe un incremento de la incidencia del compromiso hepatobiliar, probablemente secundario a una mayor sobrevida 23 : $60 \%$ de los adultos jóvenes con FQ tienen compromiso de la vesícula biliar ${ }^{24}$ pero menos de $2 \%$ desarrollan una colangitis esclerosante. La cirrosis biliar focal es característica de la FQ, se asocia con dilatación ductular y aumento del tejido fibroso; alrededor de 5\% de los adolescentes con FQ presentan cirrosis multilobular complicada con hipertensión portal. Aun cuando es raro, las várices esofágicas resultantes pueden ser el evento terminal de la enfermedad. El desarrollo de esta complicación alcanza 2\%24. Los objetivos del tratamiento de los pacientes con FQ e hipertensión portal van enfocados a una resolución pronta y vigorosa de la hemorragia gastrointestinal mediante ligadura endoscópica, shunts esplenorrenales, embolización esplénica o eventualmente trasplante hepático. Finalmente, el síndrome de obstrucción intestinal distal (DIOS: distal intestinal obstruction syndrome) es un cuadro de causa incierta que se manifiesta en forma crónica, afecta aproximadamente $5 \%$ de los pacientes con FQ y se caracteriza por una impacta- ción parcial o total de material mucoso-fecal en el íleon distal, ciego y colon distal ${ }^{25}$.

\section{OTRAS MANIFESTACIONES \\ EN EL ADOLESCENTE Y ADULTO JOVEN}

El 98\% de los hombres con FQ son infértiles debido a la azoospermia secundaria a la agenesia congénita bilateral de los conductos deferentes. Esta condición está ligada fuertemente a defectos en CFTR, pudiendo incluso manifestarse en forma única ${ }^{8,12,17}$. El potencial sexual y la espermatogénesis son normales, por lo que muchos de estos hombres pueden ser padres mediante técnicas de microaspiración espermática. La función reproductiva de la mujer es normal, pudiendo presentarse un retraso puberal respecto a la edad. Puede haber un mayor grado de deshidratación del moco cervical, lo que puede impedir la fertilidad. La prevalencia de osteoporosis en niños no llega a $1 \%$ mientras que en adultos jóvenes puede ser hasta 9\%. Esto usualmente es debido a un pobre estado nutricional, mala absorción de vitamina $\mathrm{D}$, uso de esteroides sistémicos e hipogonadismo. Las exacerbaciones pulmonares están asociadas con una aceleración de la reabsorción ósea. Sólo 34\% de los adultos con FQ tiene una densidad mineral ósea normal ${ }^{4,6}$.

\section{CONTROL Y SEGUIMIENTO DURANTE LA TRANSICIÓN}

Si bien es cierto existen muchos esquemas de manejo, sugerimos un control cada dos meses en forma ambulatoria para determinar la adherencia al tratamiento, posibles recaídas o exacerbaciones, variaciones en la función pulmonar y crecimiento, así como nuevos gérmenes en cultivos. Todos los pacientes con FQ requieren una evaluación anual clínica y de laboratorio completa, determinando los progresos durante el último año e introduciendo cambios en el tratamiento. Se debe poner énfasis en el crecimiento y desarrollo puberal, discutiendo aspectos de fertilidad en aquellos pacientes con edad apropiada. Se debe hacer una radiografia de tórax y pletismografía anualmente, así como espirometría (cada 3-6 meses) determinado la respuesta al salbutamol. Una ecografía hepática y esplénica en aquellos con pruebas de función hepática alterada (o un hígado palpable) debe ser parte de este 
control. En los pacientes en riesgo, debe considerarse una densitometría ósea anual, y si ésta es normal, repetirla cada dos años. Los exámenes de laboratorio incluyen: hemograma, electrolitos, creatinina, calcio, magnesio, fósforo, glicemia, pruebas de función hepática y de coagulación, colesterol total y fracciones, vitaminas A, D y E, IgG, A, M, E, Ig E específica para Aspergillus, que se deben repetir cada 6-12 meses dependiendo de cada sujeto. Debe revisarse las técnicas kinésicas aprendidas y la forma de aplicación de cada una de ellas.

\section{Consideraciones especiales}

La mayoría de los pacientes con FQ fallecen por insuficiencia respiratoria. Aunque la ventilación no invasiva (VNI) fue inicialmente propuesta como una conducta paliativa ${ }^{26}$, algunos estudios recientes han mostrado una mejoría del trabajo muscular incrementando la ventilación alveolar/minuto ${ }^{27}$. El gran componente inflamatorio y el aumento de la resistencia y compliance pulmonar pueden explicar el incremento en el trabajo respiratorio; sin embargo, los efectos fisiológicos de la VNI, aún son desconocidos. Se prefiere su empleo durante los estados de sueño, ya que la hipoventilación ocurre preferentemente durante el sueño REM ${ }^{28}$. Algunos pacientes experimentan una dramática mejoría en su calidad de vida. La VNI por largo tiempo, debe ser recomendada en aquellos sujetos con un claro beneficio en la sobrevida, calidad de sueño o ambos. Ya que la función pulmonar de estos pacientes experimenta, característicamente, una caída irreversible y progresiva, una disminución en la velocidad de esta caída podría representar un argumento válido para su ensayo.

Luego de más de 20 años del primer trasplante pulmonar pediátrico, la experiencia recogida no es muy alentadora; menos de 5\% de los pacientes con algún trasplante pulmonar son niños, siendo la FQ primera causa de indicación, especialmente entre los 11 y 17 años. Dependiendo del centro, la indicación para ingresar a una lista de trasplante dependerá de si la expectativa de vida oscila entre 6 y 24 meses. El promedio global de sobrevida es 3,5 años, con 54\% de sobrevida a 5 años. El rechazo del injerto es responsable del $56 \%$ de las muertes en los primeros 60 días, mientras que la bronquiolitis obliterante es responsable de $62 \%$ de las muertes tardías ${ }^{29}$. Cree- mos que el trasplante pulmonar no es la solución para la FQ, y en vista que aún no existe una “terapia estándar" para los estadios más graves, sugerimos un precoz y alto índice de sospecha (especialmente en niños), agresividad en las terapias antibióticas específicas en cada exacerbación aguda (por muy leve que ésta parezca), soporte nutricional y técnicas kinésicas permanentes para retrasar la progresión de esta enfermedad.

Transición DE LOS CuIDAdOS DEL NIÑO AL ADULTO

Todos los niños experimentan una transición en algún momento de la vida. Este término, debe ser entendido como más que una simple "transferencia" de un sitio a otro; implica un proceso, usualmente complejo, mediante el cual un individuo toma un rol activo e independiente en la sociedad, reconfigurando su actuación en la familia. Muchos de los niños que experimentan la transición lo hacen sin dificultad; sin embargo aquellos con enfermedades crónicas están sujetos a más consideraciones. Si bien los cuidados para la transición existen desde hace más de 3 décadas, aún no existen reglas fijas para su aplicación, sino más bien, sólo experiencias anecdóticas. Los programas de transición deben ser específicos para cada enfermedad. Si bien la FQ es una condición históricamente considerada "pediátrica", hoy sabemos que casi $45 \%$ de los pacientes en Europa y Norteamérica son adultos ${ }^{3,6}$; paradójicamente, una encuesta reveló que sólo 55\% de los programas de FQ en los Estados Unidos cuentan con un programa para adultos ${ }^{4,30}$.

La transición debe ocurrir en el contexto del desarrollo biológico del niño. Los cambios puberales generan muchas preguntas sobre la apariencia física, sexualidad y el significado de ser "normal". La FQ retrasa el comienzo de la pubertad y produce un menor grado de interacción y menor capacidad de socialización laboral y sentimental. La adolescencia es un periodo crítico en que ocurren numerosos cambios físicos y psíquicos; es una etapa de independencia y rebelión ${ }^{31}$, por lo que la transición debe ser flexible e individualizada; en general oscila entre los 16 y 21 años. Debe evitarse la transición si existe una inadecuada preparación y también durante las crisis respiratorias ya que puede producirse un desequilibrio psicológico ${ }^{30,32}$. 
Los sistemas de salud de adultos demandan un alto grado de responsabilidad personal y autonomía, por lo que los candidatos deberán haber demostrado habilidad en el conocimiento de sus necesidades (coordinar controles, administración de terapias, reconocer signos de deterioro respiratorio) ${ }^{33}$. Los pacientes deben conocer su propia historia de salud, tanto en aspectos generales como particulares. Se requiere una adecuada transferencia de información, lo cual implica más que un simple resumen con una larga lista de medicaciones o de exámenes. El centro receptor debe conocer el estado funcional, la progresión de la enfermedad, condiciones asociadas, complicaciones, tratamientos previos, historia nutricional, cultivos microbiológicos y sus sensibilidades, estado puberal y de fertilidad, aspectos psicosociales, expectativas de vida y la adherencia familiar ${ }^{33,34}$. Se requiere una coordinación entre el equipo de tratantes pediátricos y el equipo de adultos ${ }^{32}$. La transición es un proceso de discusión continua y programada.

Los pacientes con FQ y sus familias experimentan mucha ansiedad al abandonar las salas y policlínicos de atención pediátrica. Existe una resistencia, responsable de $45 \%$ de los fracasos ${ }^{30}$. Debe existir el concepto de programación y no de improvisación. Algunos pacientes que ya fueron transferidos participan de la bienvenida a los más jóvenes y nuevos, lo cual agrada a muchos adolescentes. Es ideal que el pediatra presente a la familia ante quien será el nuevo médico responsable, a fin de asegurar respuestas a muchos temores que aparecen en la entrevista. La transición es menos difícil si es que antes ya hubo algún contacto con el equipo de adultos. Muchas familias comentan malas experiencias con los sistemas de salud de

\section{REFERENCIAS}

1. SAWYER S. Developmentally appropriate healthcare for young people with chronic illness: questions of philosophy, policy, and practice. Pediatr Pulmonol 2003; 36: 363-5.

2. American Academy of Pediatrics; American Academy of Family Physicians; American College of Physicians-American Society of Internal Medicine. A consensus statement on health care transitions for young adults with special health care needs. Pediatrics 2002; 110: 1304-06. adultos aludiendo a hechos anecdóticos ${ }^{33}$. Existe temor de no encontrar la misma calidad de atención, o si el nuevo "escenario" será el mejor para el paciente. Es importante evaluar qué espera realmente el adolescente recibir o encontrar, ya que es evidente que el estilo de cuidado en los sistemas de adultos es diferente al estilo pediátrico.

Uno de los momentos difíciles, es definir clínicamente el estadio terminal de esta enfermedad. La valoración clínica y de laboratorio deben estar en manos de personal experimentado que pueda establecer sin dudas algunos parámetros de irreversibilidad. El eje debe ser siempre el paciente, con cuidados lo suficientemente flexibles, en relación directa con la familia. Deben evitarse los tratamientos innecesarios y cruentos, siendo la prioridad calmar el dolor y controlar los síntomas. La familia deberá estar muy bien informada y el equipo médico, receptivo a preguntas aun sin conocer las respuestas, transmitiendo comprensión y sabiduría. Se debe discutir dónde quieren (el paciente y la familia) pasar los últimos momentos de la enfermedad. Esta decisión surge muchas veces en forma espontánea en el curso de la relación médico-paciente.

En resumen, la transición del cuidado de un paciente con FQ desde la niñez a la adultez debe ser reconocida como una realidad en nuestro medio; si bien el número de casos es aún pequeño, las políticas de salud reflejadas en diversos programas nacionales, proporcionan pacientes cada vez más complejos y plantean el reto no sólo de incrementar la sobrevida, sino optimizar su calidad; para ello, se requiere un plan, comunicación y coordinación para asegurar el éxito.

3. Kulich M, Rosenfeld M, Goss $\mathrm{CH}$, Wilmott R. Improved survival among young patients with cystic fibrosis. J Pediatr 2003; 142: 631-6.

4. Dobin C, Bye P. Adults with cystic fibrosis: meeting the challenge! Intern Med J 2003; 33: 593-7.

5. Sánchez I, Pérez A, Boza L, Lezana V, Vila ma, Repetto G et al. Consenso Nacional de Fibrosis Quística. Rev Chil Pediatr 2001; 72: 356-80.

6. Cystic Fibrosis Foundation. Cystic fibrosis Foundation Patient Registry Annual Report 2000. Bethesda: Cystic Fibrosis Foundation, 2001.

7. Navarro H, Kolbach M, Repeto G, Guiraldes E, 
Harris P, Foradori A et al. Correlación genotipofenotipo de un grupo de pacientes con fibrosis quística. Rev Méd Chile 2002; 130: 475-81.

8. Ratjen F, Doring G. Cystic fibrosis. Lancet 2003; 361: 681-9.

9. Vega-Briceño LE. CFTR: 15 años después del descubrimiento de un gen. Rev Med Hered 2004; 15: 159-65.

10. Accurso F, Sontag M. Seeking modifier genes in cystic fibrosis. Am J Respir Crit Care Med 2003; 167: 289-90.

11. Gabolde M, Guilloud-Bataille M, Feingold J, Besmond C. Association of variant alleles of mannose binding lectin with severity of pulmonary disease in cystic fibrosis: cohort study. BMJ 1999; 319: 1166-7.

12. Gibson R, Burns J, Ramsey B. Pathophysiology and management of pulmonary infections in cystic fibrosis. Am J Respir Crit Care Med 2003; 168: 918-51.

13. Wilmotт R. Making the diagnostic of cystic fibrosis. J Pediatr 1998; 132: 563-5.

14. Antonelli M, Midulla F, Tancredi G, Salvatori F, Bonci E, Cimino G. Bronchial artery embolization for the management of non massive hemoptysis in cystic fibrosis. Chest 2002; 121: 796-801.

15. Flume P. Pneumothorax in cystic fibrosis. Chest 2003; 123: 217-21.

16. Tiddens H. Detecting early structural lung damage in cystic fibrosis. Pediatr Pulmonol 2002; 34: 228-31.

17. Pilewski J, Frizzell R. Role of CFTR in Airway Disease. Physiol Rev 1999; 79: 215-55.

18. Durno C, Corey M, Zielenski J, Tullis E, Tsui L-C, Durie P. Genotype and Phenotype Correlations in Patients with Cystic Fibrosis and Pancreatitis. Gastroenterology 2002; 123: 1857-64.

19. Marshall B, Butler S, Stoddard M, Moran A, Liou T, Morgan W. Epidemiology of cystic fibrosisrelated diabetes. J Pediatr 2005; 146: 681-7.

20. Walker W, Durie P, Hamilton J, Walker-Smith J, Watkins J. Pediatric Gastrointestinal Disease. 2 $2^{\mathrm{a}}$ Edición. BC Decker, Inc. Philadelphia. 1996; II p 1071-4.

21. Balistreri W. Liver disease in infancy and childhood. In: Schiff ER, Sorrell MF, Maddrey WC, eds. Schiff's diseases of the liver. Philadelphia (PA): Lippincott-Raven; 1999; p 1378-494.

22. Scott R, O'Loughinn E, Gall D. Gastroesophageal reflux in patients with cystic fibrosis. J Pediatr 1985; 106: 223-7.
23. Lenaerts C, Lapierre C, Patriquin H, Bureau N, Lepage G, Harel F et al. Surveillance for cystic fibrosis-associated hepatobiliary disease: early ultrasound changes and predisposing factors. J Pediatr 2003; 143: 343-50.

24. Lenaerts C, Lapierre C, Patriquin $\mathrm{H}$, Bureau N, Lepage G, Harel F et al. Surveillance for cystic fibrosis-associated hepatobiliary disease: early ultrasound changes and predisposing factors. J Pediatr 2003; 143: 343-50.

25. Koletzko S, Corey M, Ellis L, Spino M, Stringer DA, DuRIE PR. Effects of cisapride in patients with cystic fibrosis and distal intestinal obstruction syndrome. J Pediatr 1990; 117: 815-22.

26. Hodson M, Madden B, Steven M, Tsang V, Yacoub M. Non-invasive mechanical ventilation for cystic fibrosis patients a potential bridge to transplantation. Eur Respir J 1991; 4: 524-7.

27. Faroux B, Pigeot J, Polkey M, Isabey D, Clement A, LOFASO F. In vivo physiologic comparison of two ventilators used for domiciliary ventilation in children with cystic fibrosis. Crit Care Med 2001; 29: 2097-105.

28. Milross M, Piper A, Norman M, Becker H, Willison G, Grunstein R. Low-flow oxygen and bilevel ventilatory support: effects on ventilation during sleep in cystic fibrosis. Am J Respir Crit Care Med 2001; 163: 129-34.

29. Mallory G, Spray T. Paediatric lung transplantation. Eur Respir J 2004; 24: 839-45.

30. Flume P, Anderson D, Hardy K, Gray S. Transition programs in cystic fibrosis centers: perceptions of pediatric and adult program directors. Pediatr Pulmonol 2001; 31: 443-50.

31. Sawyer S, Rosier M, Phelan P, Bowes G. The selfimage of adolescents with cystic fibrosis. J Adolesc Health 1995; 16: 204-8.

32. Zack J, Jacobs C, Keenan P, Harney K, Woods E, Colin A, Emans S. Perspectives of patients with cystic fibrosis on preventive counselling and transition to adult care. Pediatr Pulmonol 2003; 36: 376-83.

33. Magde S, Bryon M. A model for transition from pediatric to adult care in cystic fibrosis. J Pediatr Nurs 2002; 17: 283-8.

34. Olveira C, Pérez-Ruiz E, Pérez-Frías J, Martin J, Domenech A, Valencia A. Transition from pediatric cystic fibrosis units to adult units. Arch Bronconeumol 2001; 37: 444-8. 